\title{
Op-Ed
}

\section{Treating depression in patients with chronic disease}

\author{
Recognition and treatment are crucial; depression worsens the course of a chronic illness see also p 332
}

Gregory E Simon
Center for Health
Studies
Group Health
Cooperative
1730 Minor Ave, Ste
1600
Seattle, WA 98101-1448
simon.g@ghc.org
Funding: Supported by
NIMH grant MH51338
Competing interests:
None declared
West J Med
2001;175:292-293

2001;175:292-293
Chronic medical illness is consistently associated with an increased prevalence of depressive symptoms and disorders. $^{1,2}$ In some cases, depression appears to result from specific biologic effects of chronic medical illness. Examples of this relationship include central nervous system disorders—such as Parkinson's disease, cerebrovascular disease, or multiple sclerosis-as well as endocrine disorders - such as hypothyroidism. In other cases, the association between depression and chronic medical illness appears to be mediated by behavioral mechanisms; the limitations on activity imposed by the illness lead to gradual withdrawal from rewarding activities. ${ }^{3}$ Why should primary care physicians be alert to the possibility of depression in their patients with chronic disease? Why do they sometimes miss it? And what can they do to manage this distressing mental health problem?

Depression significantly increases the overall burden of illness in patients with chronic medical conditions. Compared with those without depression, medical outpatients with depressive symptoms or disorders experienced decrements in quality of life ${ }^{4}$ and had almost twice as many days of restricted activity or missed work because of illness. ${ }^{5}$ Similarly, depression is associated with a $50 \%$ to $100 \%$ increase in health services use and costs. ${ }^{6}$

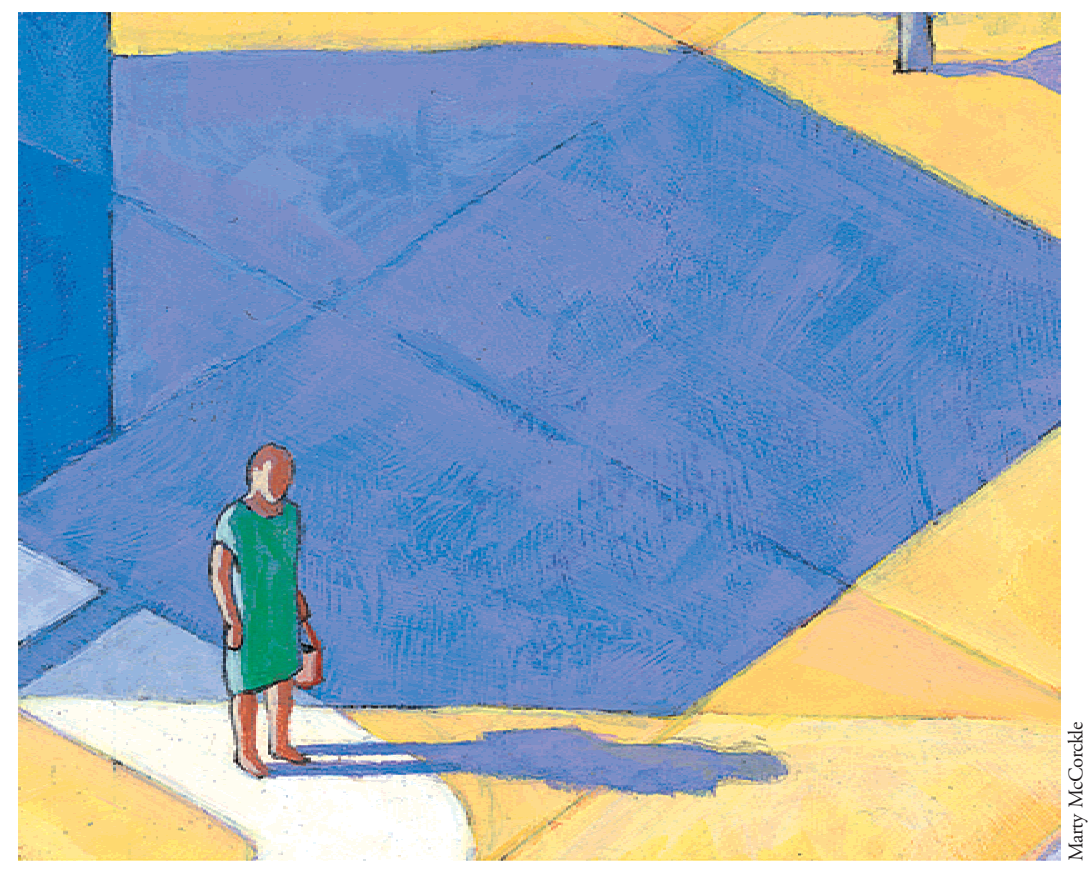

Depression adds to the burden of illness in patients with chronic disease
Depression has also been linked to increased diseaserelated morbidity and mortality. Results of populationbased studies have shown a modest association between depression and all-cause mortality and a stronger association between depression and mortality resulting from cardiovascular disease. ${ }^{7}$ Depression is clearly associated with a poorer prognosis and more rapid progression of chronic illnesses, including ischemic heart disease ${ }^{8}$ and diabetes. ${ }^{9}$ Here as well, the interaction between depression and chronic medical illness may be mediated by either biologic or behavioral mechanisms. For example, depression may affect the course of ischemic heart disease through increased platelet activation or of diabetes through decreased glucose tolerance. It may also affect these diseases by decreasing treatment adherence and physical activity and by increasing tobacco and alcohol use. ${ }^{10}$

The presence of a chronic medical illness may reduce the likelihood that physicians or other health care providers recognize or treat depression. The demands of chronic illness management may crowd concerns of depression out of the visit agenda. Providers may also not look beyond a chronic medical illness to explain nonspecific symptoms, such as fatigue or poor concentration. Even when they recognize symptoms of depression, they may defer treatment, believing that "anyone would be depressed" in such a situation.

Yet, somatic symptoms often reflect a combination of medical and psychological factors, and the presence of a clear medical explanation for these symptoms does not rule out depression as a contributing factor. ${ }^{11}$ Patients should receive appropriate treatment regardless of whether there is an obvious medical or psychological precipitant to their depression.

Because the symptoms of depression — such as fatigue or changes in appetite-may overlap with manifestations of the medical illness, using standard screening tools or diagnostic criteria for depression in patients with chronic disease may lead to overdiagnosis of depression. This concern has led to the development of depression measures, such as the Geriatric Depression Scale, ${ }^{12}$ that include fewer somatic symptoms. However, it seems that underdiagnosis of depression is a more important problem than is overdiagnosis. Concern about the accuracy of screening or diagnostic measures should not interfere with efforts to improve access to appropriate treatment.

So what is the appropriate treatment? First, consider whether a medical problem or medication is causing the 
depressive symptoms; treating the problem or changing the medication may alleviate the symptoms. Chronic illnesses known to mimic depression include sleep apnea, thyroid disorders, and Cushing's syndrome. Among medical outpatients, however, the prevalence of major depression $(6 \%-10 \%)$ is greater than that of "medical mimics" of depression. Exhaustive efforts to rule out medical disorders can delay needed treatment and reinforce the stigmatization of depression as something less than a "real" illness.

Second, recognize that randomized trials have shown the efficacy of both pharmacologic ${ }^{13-15}$ and psychosocial ${ }^{16}$ treatments of depression across a range of chronic medical conditions. Effective treatment of depression reduces depressive symptoms and improves daily functioning. Treating depression has also been shown to have a positive effect on biologic indicators of disease severity or progression, such as the level of glycosylated hemoglobin in diabetes $^{15}$ or platelet activation in ischemic heart disease. ${ }^{17}$ Given the clear benefits of recognizing and treating depression, all those who care for people with chronic medical illness should consider identification and treatment of depression a clinical priority.

References

1 Wells K, Golding JM, Burnam MA. Psychiatric disorder in a sample of the general population with and without chronic medical conditions. Am J Psychiatry 1988;145:976-981.

2 Anderson R, Freedland K, Clouse R, Lustman P. The prevalence of comorbid depression in adults with diabetes: a meta-analysis. Diabetes Care 2001;24:1069-1078.

3 Prince M, Harwood R, Thomas A, Mann A. A prospective population-based cohort study of the effects of disablement and social milieu on the onset and maintenance of late-life depression. The Gospel Oak Project VII. Psychol Med 1998;28:337-350.
4 Spitzer R, Kroenke K, Linzer M, et al. Health-related quality of life in primary care patients with mental disorders. Results from the PRIME-MD 1000 Study. JAMA 1995;274:1511-1517.

5 Ormel J, VonKorff M, Ustun TB, Pini S, Korten A, Oldehinkel T. Common mental disorders and disability across cultures. Results from the WHO Collaborative Study on Psychological Problems in General Health Care. JAMA 1994;272:1741-1748.

6 Simon G, VonKorff M, Barlow W. Health care costs of primary care patients with recognized depression. Arch Gen Psychiatry 1995;52:850-856.

7 Unutzer J, Patrick D, Marmon T, Simon G, Katon W. Depressive symptoms and mortality in a prospective study of 2,558 older adults. Am J Geriatr Psychiatry; in press.

8 Glassman A, Shapiro P. Depression and the course of coronary artery disease. Am J Psychiatry 1998;155:4-11.

9 de Groot M, Anderson R, Freedland K, Clouse R, Lustman P. Association of depression and diabetes complications: a meta-analysis. Psychosom Med 2001;63:619-630.

10 DiMatteo M, Lepper H, Croghan T. Depression is a risk factor for noncompliance with medical treatment: meta-analysis of the effects of anxiety and depression on patient adherence. Arch Intern Med 2000;160:2101-2107.

11 Pearson S, Katzelnick D, Simon G, Manning W, Helstad C, Henk H. Depression among high utilizers of medical care. J Gen Intern Med 1999; 14:461-468.

12 Yesavage J, Brink TL, Rose TL, et al. Development and validation of a geriatric depression screening scale. J Psychiatr Res 1982-1983;17:37-49.

13 Roose S, Laghrissi-Thode F, Kennedy J, et al. Comparison of paroxetine and nortriptyline in depressed patients with ischemic heart disease. JAMA 1998;279:287-291.

14 Borson S, McDonald G, Gayle T, Deffebach M, Lakshminarayan S, VanTuinen C. Improvement in mood, physical symptoms, and function with nortriptyline for depression in patients with chronic obstructive pulmonary disease. Psychosomatics 1992;33:190-201.

15 Lustman P, Freedland K, Griffith L, Clouse R. Fluoxetine for depression in diabetes: a randomized double-blind placebo-controlled trial. Diabetes Care 2000;23:618-623.

16 Sharpe L, Sensky T, Timberlake N, Ryan B, Brewin C, Allard S. A blind, randomized, controlled trial of cognitive-behavioural intervention for patients with recent onset rheumatoid arthritis: preventing psychological and physical morbidity. Pain 2001;89:275-283.

17 Pollock B, Laghrissi-Thode F, Wagner W. Evaluation of platelet activation in depressed patients with ischemic heart disease after paroxetine or nortriptyline treatment. J Clin Psychopharmacol 2000;20:137-140. 